\title{
Erratum to: An economic analysis of voting in Sweden
}

\section{Henrik Jordahl}

Published online: 27 October 2011

(C) Springer Science+Business Media, LLC 2011

Erratum to: Public Choice (2006) 127:251-265

\section{DOI 10.1007/s11127-006-0864-5}

Equations (3), (4), and (5) are incorrect in the published article. The correct equations are as follows:

$$
\begin{aligned}
\operatorname{Pr}(\mathrm{VOTE}=1)= & \Phi\left[a+G\left(b_{1} \Delta U+b_{2} \Delta I+c_{1} \mathrm{MICRO}\right)\right. \\
& \left.+c_{2} \mathrm{UNEMPLOYED}+d\right], \\
\operatorname{Pr}(\mathrm{VOTE}=1)= & \Phi\left[a+G\left(b_{1} \Delta U+b_{2} \Delta I+b_{3} \Delta \mathrm{GDP}+c_{1} \mathrm{MICRO}\right)\right. \\
& \left.+c_{2} \mathrm{UNEMPLOYED}+d\right], \\
\operatorname{Pr}(\mathrm{VOTE}=1)=\Phi\left[a+G\left(b_{1} \mathrm{MACRO}+c_{1} \mathrm{MICRO}\right)\right. & \\
& \left.+c_{2} \mathrm{UNEMPLOYED}+d\right] .
\end{aligned}
$$

The online version of the original article can be found under doi:10.1007/s11127-006-0864-5. 